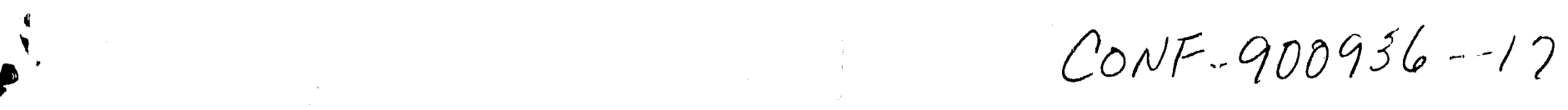

"The submitted manuscript has been authored by a contractor of the U.S. Government under contract No. DEAC05-840R21400. Accordingly, the CONF-900936--17 U.S. Government retains a nonexclusive, royalty-free license to publish or reproduce the published form of this contribution, or allow others to do so, for U.S. Government purposes."

\title{
ION-INDUCED DAMAGE AND AMORPIIZATION IN Si
}

O. W. Holland and C. W. White, Oak Ridge National Laboratory, Oak Ridge, TN 37831

\section{Abstract}

Ion-induced damage growth in high-energy, self-ion irradiated Si was studied using electron microscopy and Rutherford backscattering spectroscopy. The results show that there is a marked variation in the rate of damage growth, as well as the damage morphology, along the path of the ion. Near the ion end-of-range (eor), damage increases monotonically with ion fluence until a buried amorphous layer is formed, while damage growth saturates at a low level in the region ahead. The morphology of the damage in the saturated region is shown to consist predominantly of simple defect clusters such as the divacancy. Damage growth remains saturated ahead of the eor until expansion of the buried amorphous layer encroaches into the region. A homogeneous growth model is presented which accounts for damage saturation, and accurately predicts the dose-rate dependence of the saturation level. Modifications of the model are discussed which are needed to account for the rapid growth in the eor region and near the interface of the buried amorphous layer. Two important factors contributing to rapid damage growth are identified. Spatial separation of the Frenkel defect pairs (i.e interstitials and vacancies) due to the momentum of the interstitials is shown to greatly impact damage growth near the eor, while uniaxial strain in the interfacial region of the amorphous layer is identified as an important factor contributing to growth at that location.

\section{DISCLAIMER}

This report was prepared as an account of work sponsored by an agency of the United States Government Neither the United States Governm nt nur any agency thereof, nor any of their Coress or mplied, or assumes any legal liability or responsi employees, makes any wranty, express or implied, of assumes any legat hability or responsi- or bility for the accuracy, completeness, or usefulness of any information, apparatus, product, process disclosed, or represents that its use would not infringe privately onined rights. Reference herein to any specific commercial product, process, or service by trade name, trademark. manufacturer, or otherwise does not necessarily constitute or imply its endorsement, recommendation, or favoring by the United States Covernment or any agency thereof. The vinions of authors expressed herein do not necessarily state or reflect those of the United States Government or any agency thereol. 


\section{Introduction}

Damage accumulation in $\mathrm{Si}$ during ion irradiation remains an active area of research, with many aspects still not well understood. This, however, has not prevented implantation from becoming a well-established processing technique in IC fabrication for controlled doping of $\mathrm{Si}$. In this regard, the damage associated with implantation is undesirable and must be removed before suitable dopant activation and carrier mobility are achicved. Annealing cycles required to achieve the desired electrical properties are generally determined empirically. However, new innovative uses of ion implantation have been proposed which can be categorized as "defect engineering" in which a desired property of $\mathrm{Si}$ is achieved by the controlled introduction of ion-induced damage. Applications of defect engineering are found in damage annealing where it has been used to reduce secondary defect formation during implantation, 1,2 and in the production of damaged layers for

impurity gettering. ${ }^{3-5}$ Clearly, the ability to manipulate the damage morphology and its stability are critical to the success of such engineering of material properties. This ability derives from a knowledge of damage nucleation and growth processes during ion irradiation in $\mathrm{Si}$ and, therefore, provides motivation for such studies.

The results of high-energy, self-ion irradiation in $\mathrm{Si}$ single crystal at room temperature (RT) are reported. Reasons for using self-ions are twofold; first the effects of chemically dissimilar ions on damage formation are eliminated, and second a broader understanding of damage growth mechanisms for different ions is achieved. The latter reason arises because knowledge of self-ion collision cascades can be used to understand subcascade regions (formed by energetic knockon atoms) which are a fundamental component of any high-energy ion cascade. Investigations were concerned with both characterization of the ion-induced damag, and determination of nucleation and srowth mechanisms. Both ransmission electron microscopy (TEM) and Rutherford backscattering/channeling spectrose spy (RBS) were used in characterizing the damage. The growth and morpho'ogy of the dan age are shown to vary markedly over the range of the ions. 
The different behaviors are identified, and the nature of the defect interactions which give rise to these differences are detailed. This study provides new insight into damage growth mechanisms, especially those responsible for amorphization in $\mathrm{Si}$, and demonstrates the benefits of using of high-energy ions isi such investigations. Not only do high energy ions offer the possibility of new irradiation effects but the extended path length of the ions allows subtle, and sometimes not so subtle, changes in phenomena over the path length of the ions to be more easily studied.

\section{Experimental Procedure}

Czochralski-grown $p$-type $\operatorname{Si}(100)$ wafers with resistivities between 6 and $8 \Omega$-cm were used in the study. Samples were implanted with a raster-scanned beam of ${ }^{28} \mathrm{Si}^{+}$ions from a $1.7 \mathrm{MeV}$ tandem accelerator. An energy of $1.25 \mathrm{MeV}$ and an average current density of $\sim 0.10$ $\mu \mathrm{A} / \mathrm{cm}^{2}$ were used, unless otherwise specified. The use of a tandem accelerator ensures that the ${ }^{28} \mathrm{Si}^{+}$beam will be free of contaminants, such as molecular ions of the same mass-to-charge ratio, as might be present using a single-ended machine. Structural characterization of cross-sectionally thinned samples was done using a Phillips EM400T transmission electron microscope. Damage profiles were measured by $\mathrm{RBS} /$ channeling techniques using $2.75 \mathrm{MeV} \mathrm{He}{ }^{+}$ions.

\section{Results and Discussions \\ 3.1. Damage Characterization}

Ion channeling spectra are shown in Fig. 1 from samples irradiated at RT with $1.25 \mathrm{MeV}$ $\mathrm{Si}^{+}$self-ions over a fluence range spanning an order of magnitude $f . .10 .8 \times 10^{15}$ to $8.0 \times$ $10^{15} / \mathrm{cm}^{-2}$. The spectra are numbered to indicate increasing fluen. it is clear that the rate of damage accumulation during irradiation varies widely over the range of the ions (as indicated by the aligned scattering yield). Damage near the ions' end-of-range (eor), at $\sim 1.3 \mu$, increases monotonically with dose until amorphization occurs. This is reflected in spectrum \#5 by the 
random scattering yield from this region. Grcwth of the eor damage is similar to that observed during light-ion irradiation of Si at substantially lower energies $(<2.00 \mathrm{keV})$ where two distinct growth regimes have been identified; 6-8 an initial regime during which damage accumulates slowly with a sublinear dependence on dose, followed by superlinear growth leading to amorphization. In contrast to the eor behavior, damage growth ahead of the eor and extending to the surface is seen to be absent. Damage in this region remains constant at a saturation level well below that of arnorphous Si. The approach to damage saturation is shown in Fig. 2 by the dose dependence of the minimum yield from a region near the surface. Damage initially increases linearly with dose but saturates by a fluence of $\sim 10^{15} \mathrm{~cm}^{-2}$. Saturation of damage persists during irradiation until this region is encroached upon by the expansion of the buried amorphous layer. The influence of the amorphous-crystal $(a-c)$ interface on damage accumulation in the $c$-Si adjacent to the interface is discussed later.

TEM micrographs in Fig. 3 reveal the microstructure in two samples implanted at different fluences; one with a fluence of $0.25 \times 10^{16} \mathrm{~cm}^{-2}$ (Fig. 3a) and the other with $10^{16} \mathrm{~cm}^{-2}$ (Fig. 3b). In both micrographs, the top $1 \mu$ layer is seen to be essentially void of extended crystalline defects. This layer corresponds to the region of saturated growth, as discussed above. Studies showed the annealing behavior of the damage in this region to be very similar to that associated with the divacancy defec? 9,10 Both the annealing and the microscopy results strongly suggest that the divacancy and/or small defect clusters are formed predominantly during saturated damage growth. There is no evidence of amorphization in the micrograph of Fig. 3a; only small, loop-like interstitial defects can be identified near the eor. However, a buried arıorphous layer is formed at the higher fluence, as clearly seen in Fig. 3b. This layer has distinct but undulated interfaces, and defects similar to the eor defects (i.e. interstitial loops) are observed near the interfaces. The formation of these interstitial-type defects in regions (near the eor and the $a-c$ interface) where amorphization occurs, suggests that they are an important prec.irsor to this phase 
transition. A more detailed model of how the formation of these defects influences the $c-a$ transition is given in Ref. (6).

\subsection{Homogeneous Damage Growth Model}

Damage saturation ahead of the eor can be explained by a homogeneous damage growth model. It is assumed in this model that point defects created along an ion track are sufficiently long-lived that they become uniformly distributed within the irradiated volume, where they react with defects created by other ion cascades. The ability of this model to account for damage growth in light-ion irradiated $\mathrm{Si}$ has been previously demonstrated, 11,12 although the model's validity in the present case must be judged by its ability to explain observed phenomena and to make verifiable predictions. Chemical rate equations are used to express the defect reacituns which are empirically known to consist of simple ones such as väcancy-interstitial recombination, vacancy clustering, annealing (such as interstitial-divacancy reaction), and possibly interstitial clustering. Rate equations for each of the defect species present in the reaction volume are given as follows;

$$
\begin{aligned}
& \frac{d n_{i}}{d t}=G-k_{i} n_{i} n_{v}-k_{2} n_{i} n_{o}^{v}-2 k_{4} n_{i}^{2}+k_{5} n_{v} n_{o}^{i} \\
& \frac{d n_{v}}{d t}=G-k_{i} n_{i} n_{v}+k_{2} n_{i} n_{o}^{v}-2 k_{3} n_{v}^{2}-k_{5} n_{v} n_{o}^{i} \\
& \frac{d n_{o}^{v}}{d t}=k_{3} n_{v}^{2}-k_{2} n_{i} n_{o}^{v} \\
& \frac{d n_{0}^{i}}{d t}=k_{4} n_{i}^{2}-k_{5} n_{v} n_{o}^{i}
\end{aligned}
$$

where $\mathrm{G}$ is the generation rate for vacancies and interstitials, assumed initially to be the equal. The defect concentrations are given by the $n$ values, where $n_{\mathrm{i}}, n_{\mathrm{v}}, n_{\mathrm{O}}^{\mathrm{v}}, n_{\mathrm{O}}^{\mathrm{i}}$ are the concentration of the interstitial, vacancy, divacancy, and diinterstitial, respectively. The reaction rates represented by 
the $k$ 's can be quite complex because of their dependence on both the configuration of the defects (i.e. geometrical effects) and their charge-state. The complete solution of these equations is somewhat complicated, but is not needed to understand its basic behavior. Rather, it is only necessary to realize that detailed balancing of the defect reactions yields a steady-state solution with stationary values $\left(\sim \mathrm{G}^{1 / 2}\right)$ for the different defect populations. This steady-state condition clearly can account for damage saturation observed in self-ion irradiated Si. Figure 4 shows aligned spectra from samples which were implanted identically $\left(1.25 \mathrm{MeV}, 1 \times 10^{16} \mathrm{~cm}^{-2}\right)$ but at different dose-rates. it is clear that the saturation level (as indicated by the aligned yield ahead of the buried amorphous layer) is lower in the sample implanted at the smaller dose-rate, consistent with the dose-rate dependent stationary values predicted by this model. Extraction of the total damage concentration from the two spectra yielded a $\sim 1 / 3$ power dependence on dose-rate which is somewhat smaller than the model value. This may be due to beam heating effects at the higher dose-rate (i.e. higher input power) or a nonlinear scaling between defect concentration and ion scattering yield. Nonetheless, the existence of a dose-rate dependent saturation level provides confirmation of this model. It is interesting to note the dose-rate dependence of the eor damage (as indicated by the thicker buried amorphous layer formed at the higher dose-rate). Such effects have been previously observed at lower ion energies. 12

It is of interest to examine conditions under which the steady state solution becomes untenable. The goal is to understand the nature of the defect interactions in the eor region and in the vicinity of the $a-c$ interface where unconstrained damage growth occurs. Implicit in this anal, sis is the assumption that growth is homogeneous and differences in the growth behavior are due to factors which affect the defect interactions. Clearly, this assumption must be examined before continuing. First, growth in the region of damage saturation has already been shown to be homogeneous, and encroachment upon this region by the advancing $a-c$ interface is not expected to change this basic growth mechanism. Rather, the a-c interface is thought to affect or alter the defect reactions in its proximity to produce the rapid damage growth. However, the validity of a 
homogeneous growth mechanism near the eor is not as apparent and requires further analysis. In Brinkman's description of coll'sion cascades, he argued that coliective effects can occur when the mean free path of the ion between displacement events is of the order of the interatomic spacing in the lattice. ${ }^{13}$ Such a condition in a solid is referred to as a displacement cascade and is approached for self-ion irradiation in Si only at low ion energies, $\sim 1 \mathrm{keV}$. Brinkman proposed that the collective motion (which consists of an outward flux of interstitials from the cascade volume) could nucleate damage during quenching of the cascade. Other collective effects associated with collision cascades with high-energy density $\left(\sim 1 \mathrm{eV} /\right.$ atom) have also been reported. ${ }^{14-16}$ This critical energy density is achieved for self-ions in Si at energies similar to those which satisfy Brinkman's condition. Clearly, such mechanisms are quite different from homogeneous growth and, at first, may seem capable of contributing to damage growth near the eor of the ions. However, it must be realized that eor conditions for a self-ion are identical to those for a knockon atom with comparable energy. Any nucleation mechanism associated with quenching of high-density cascades of selfions near their eor would also occur for energetic knockon atoms. Calculated values 17 of the energy transfer during scattering between a $1.25 \mathrm{MeV}$ self-ion and a lattice atom in Fig. 5 show that transfer of $>1 \mathrm{keV}$ occurs with a probability of $2.7 \times 10^{-3}$. At this value, $\sim 10$ knockons are created within this energy range by an ion in penetrating $1 \mu$ in $\mathrm{Si}$. This estimate is actually too small since the transfer probability increases as the ion losses energy over its path. Therefore, the density of such knockons can become quite large in the region of saturation, especially at the highest dose where saturation persists. The absence of discrete cascade effects associated with damage growth ahead of the eor, despite the high density of energetic knockons in this region, suggests that these effects do not contribute to damage growth in any region, and therefore, provides justification for the assumption of homogeneous growth.

It is now possible to return to the examination of the rate equations. The simplest modification which impacts on their behavior is to assign unequal generation rates for interstitials and vacancies. Since these defects are created in pairs during Frenkel defect production, the 
results in a high concentration of interstitial defects just beyond the eor while excess vacancies occur in the region ahead.

In the above example, the excess defect concentrations produced by the two effects (implantation and spatial separation of the Frenkel pairs) are of the same order of magnitude. As such, it tends to deemphasize the importance of the Frenkel separation mechanism. A dramatic and more convincing example of this mechanism's impact on damage growth can be seen in Fig. 8. A cross-sectional, TEM micrograph in Fig. 8b shows the damage morphology in a Si crystal after implantation with $10^{16} \mathrm{As}^{+} / \mathrm{cm}^{2}$ at an energy of $100 \mathrm{keV}$ and a flux of $185 \mu \mathrm{A} / \mathrm{cm}^{2}$. Substantial beam heating of the sample at :his high flux prevents amorphization but instead results in the formation of a unique damage morphology. A band containing a high density of voids, whose average size is $5 \mathrm{~nm}$, is formed in the top $45 \mathrm{~nm}$ region. Below this is a band of interstitial-type, dislocation tangles extending to $\sim 0.2 \mu$. The results of TRIM simulation of the excess defects produced by these conditions is shown in Fig. 8 a on a depth scale which matches the scale in the micrograph. It should be noted that the peak of the implanted As profile (not shown) corresponds to a concentration of $1.6 \times 10^{21} \mathrm{As} / \mathrm{cm}^{3}$ which is substantially less than the peak concentrations in the figure $\left(\sim 10^{22} \mathrm{~cm}^{-3}\right.$ for vacancies and $\sim 5 \times 10^{21} \mathrm{~cm}^{-3}$ for interstitials). The TRIM simulation essentially replicates both the damage distribution and it's basic morphology. The region of excess vacancies (extending from the surface to $50 \mathrm{~nm}$ ) corresponds almost exactly with the distribution of voids in the sample, while the region of excess :nterstitials closely matches the band of interstitial dislocations. Clearly, damage growth and its morphology are to a large extent controlled by the mechanism of Frenkel defect separation by momentum transfer to interstitials (under these irradiation conditions). 


\subsection{Defect Interaction in the Vicinity of the a-c Interface}

The effect of the $a-c$ interface on damage growth in the adjacent $c$-Si region is now examined. A buried amorphous layer was formed in (100) oriented Si by implantation with $100 \mathrm{keV}$ self-ions at a fluence of $4.5 \times 10^{14} \mathrm{~cm}^{-2}$. These conditions produce a buried layer with a distinct front interface sufficiently cluse to the surface to be easily investigated by ion channeling spectroscopy. Channeling spectra along $<110>$ from this sample are shown in Fig. 9 together with a random spectrum. Two different techniques were used to align the sample; a "substrate aligned" technique which utilizes a defect-free region behind the buried $a$-layer to orient the sample, and a "surface alignment" techrique which uses the $c$-region ahead of the buried layer. While both aligned spectra indicate the presence of a buried amorphous layer extending to $0.17 \mu$, distinct differences between the spectra are seen in the front interfacial region of the buried layer. No such differences were observed in spectra (not shown) aligned along the normal $<100>$ axis. These observations are explained by the fiesence of uniaxial strain at the interface ${ }^{18-20}$ (directed normal to the surface). The spectral differences occur only in a limited range indicating that the strain field extends only $\sim 5 \mathrm{~nm}$ ahead of the interface. The magnitude of the strain is determined by comparing the angular scan!: about $<110>$ in Fig. 10 from strained and unstrained regions of the lattice. The minimum of the scan curves clearly do not occur at the same angle, but are displaced by, $\delta \theta=0.10^{\circ}$. This yields a positive value of $0.35 \%$ for the strain, as determined by the expression $\sim-2 \tan \delta \dot{\theta}$. The interaction of point defects with the strain field is considered to be a significant factor contributing to the damage growth in the interfacial region. It is well known that interstitials are attracted to regions of dilation and, as such, are gettered by a positive strain field. While this gettering leads to damage growth as discussed pre' ously, it can also favor reactions such as interstitial clustering. The presence of interstitial-type of dislocations in the vicinity of the interface is considered to be evidence of this clustering, and also provides support for this proposed interfacial growth mechanism. 


\section{Conclusions}

Ion-induced damage in Si during high-energy, self-ion irradiation was studied. The behavior of the damage growth was shown to vary markedly over the range of the ions, from rapid growth near the eor to damage saturation in the region ahead. A comprehensive model was proposed to account for the details of damage growth over the entire ion range. The model incorporated a homogeneous nucleation and growth mechanism to explain damage saturation, and the dose-rate dependence of the saturation level. Modifications to the model to account for unconstrained damage growth near the eor and at the $a-c$ interface were discussed. Properties of these regions were examined to provide justification for these modifications. This resulted in the identification of conditions, heretofore not known or fully appreciated, which greatly impact on the behavior of damage growth. Excess atoms near the eor were identified as an important factor in determining growth behavior in this region. Both the implanted specie and spatial separation of Frenkel defect pairs were shown to contribute to this excess. Dramatic and convincing evidence was given to establish Frenkel pair separati $\mathrm{n}$ as an important, and possibly dominant, mechanism contributing to damage growth. Rapid growth near the a-c interface was tentatively attributed to the effects of an uniaxial strain field found to be localized within $\sim 5 \mathrm{~nm}$ of the interface. Interstitial gettering by this strain field was suggested as the mechanism contributing to the growth.

\section{Acknowledgements}

The authors would like to acknowledge stimulating discussions with J.H. Barrett which helped guide us through this work. Also, we would like to thank M. K. El-Ghor and J. Narayan for microscopy support. Research was sponsored by the Division of Materials Sciences, U. S. Department of Energy, under contract DE-AC05-84OR21.400 with Martin Marietta Energy Systems, Inc. 
generation rates are generally assumed to be equal. However, irradiation conditions exist (to be discussed later) in which the "effective" rates are different. An excess of one type of defect clearly will exclude steady state conditions and lead to unconstrained damage growth. Another possibility is the presence of reactions involving sinks, which preferentially getter one type of the point defect, and thus allow the complimentary defect and its complexes to increase without constraint. Therefore, only simple modifications of the homogeneous model are needed to account for the different growth behaviors observed within the irradiated region. The eor and $a-c$ interfacial regions will now be examined more carefully to see if these conditions provide any insight into growth of damage in these regions.

\subsection{Defect Interactions near End-of-Range}

TRIM 17 computer simulation in Fig. 6 shows both the density of Frenkel defects along the ion track (normalized to $10^{15}$ ions $/ \mathrm{cm}^{2}$ ) and the implanted ion profile. Clearly, there is little correlation between the distribution of Frenkel defects and the damage distribution near the eor as indicated by the ion scattering results in Fig. 1. This occurs despitr the fact that the Frenkel density varies only $\sim 30 \%$ between the eor region (where monotonic growth is observed to occur) and the adjacent region where damage growth is saturated. Rather, the damage profile more closely resembles the implant distribution indicating that parameters associated with this distribution, other than deposited energy density, are critical in determining the behavior of the eor damage growth. The most obvious effect of the implant distribution on the conditions near the eor is that it contributes excess atoms to the region, $r^{-}$.ny of which end up as interstitial defects. This situation is further enhanced as a result of the energy transfer between the ion and target atoms which ensures that knockon atoms have an average, non-zero component of momentum along the incident direction of the ions. This creates a spatial separation of the Frenkel defect components, especially near the eor. Both effects are seen in the results of TRIM simulation in Fig. 7 for $300 \mathrm{keV} \mathrm{Si}{ }^{+}$self-ion irradiation. The spatial separation of the the Frenkel defect components 


\section{References}

1. W.X. Lu, Y.H. Qian, R.H. Tian, Z.L. Wang, R.J. Schreutelkamp, J.R. Liefting, and F.W. Saris, Appl. Phys. Lett. 55, 1838 (1989).

2. W.X. Lu, R.J. Schreutelkamp, J.R. Liefting, and F.W. Saris, "Reduction of Preamorphization Damage by Multiple Implantation Prior to Annealing", these proceedings.

3. H.Wong, N.W. Cheung, P.K. Chu, J. Liu, and J.W. Mayer, Appl. Phys. Lett. 52, 1023 (1988).

4. M. Tamura, T. Ando, and K. Ohyu, "MeV Ion-Induced Damage in Si and Its Annealing", these proceedings.

5. K. Tsukamoto, S. Komori, T. Kuroi, and Y. Akasaka, "High Energy Ion Implantation for ULSI", these proceedings.

6. O.W. Holland, S.J. Pennycook, and G.L. Albert, Appl. Phys. Lett. 55, 2503 (1989).

7. F.H. Eisen and B. Welch, in Proceedings of the European Conference on Ion Implantation (Peter Peregrinus, Reading, 1970), p. 227.

8. F.H. Eisen and B. Welch, Radiat. Eff. 7, 143 (1971).

9. H.J. Stein, F.L. Vook, and J.A. Borders, Appl. Phys. Lett. 14, 328 (1969).

10. L.J. Cheng, J.C. Corelli, J.W. Corbett, and G.D. Watkins, Phys. Rev. 152, 761 (1966). 
i1. O.W. Holland, M.K. El-Ghor, and C.W. White, Appl. Phys. Lett. 53, 1202 1988).

12. O.W. Holland, D. Fathy, J. Narayan, and O.S. Oen, Radiat. Eff. 90, 127 (1985).

13. J.A. Brinkman, J. Apnl. Phys. 25, 961 (1954).

14. L.A. Thompson, Radiat. Eff. 56, 105 (1981) and references within.

1: Y.-T. Cheng, M. V'an Rossum, M.-A. Nicolet, and W.L. Jchnson, Appl. Phys. Lett. 45, 185 (1984).

16. Y.-T. Cheng, M.-A. Nicolet, and W.L. Johnson, Phys. Rev. Lett. 58, 2083 (1987).

17. J.P. Biersack and L. G. Haggmark, Nucl. Instrum. Methods, 174, 257 (1980).

18. O.W. Holland, J.D. Budai, and C.W. White, Appl. Phys. Lett. 57, 243 (1990).

19. L.C. Feldman, J. Bevk, B.A. Davidson, H.-J. Gossmann, and J. P. Mannaerts, Phys. Rev.Lett. 59, 664 (1987).

20. B.C. Larson, C.W. White, and B.R. Appleton, Appl. Phys. Lett. 32, 801 (1978). 


\section{FIGURE CAPTIONS:}

Fig. 1. $<100>$ aligned spectra from $\mathrm{Si}(100)$ samples implanted at various fluences with $1.25 \mathrm{MeV}$ self-ions.

Fig. 2. Dose dependence of the minimum yield in Si implanted with $1.25 \mathrm{MeV}$ self-ions.

Fig. 3. Cross-sectinnal, TEM micrographs of Si samples implanted with $1.25 \mathrm{MeV}$ self-ions at a fluence of (a) $0.25 \times 10^{16} \mathrm{~cm}^{-2}$ and (b) $10^{16} \mathrm{~cm}^{-2}$.

Fig. 4. Comparison of $\langle 100\rangle$ aligned spectra from Si crystals which were implanted at different dose-rates.

Fig. 5. Energy transfer vs. impact parameter for scattering of a self-ion by a Si atom calculated for two ion energies, $1.25 \mathrm{Mev}$ and $100 \mathrm{keV}$. The interatomic potential and the calculation method are described in ref. (17). Probability of an energy transfer exceeding a given value is determined simply as the square of the ratio between the impact parameter (corresponding to the energy transfer), and half the average interatomic spacing in the lattice.

Fig. 6. TRIM calculation of the depth distribution of Frenkel pairs and implanted specie after implantation of Si with 1,25 MeV self-ion at a fluence of $10^{15} \mathrm{~cm}^{-2}$.

Fig. 7. TRIM calculation of the distribution of implanted specie, and excess interstitial defects in Si irradiated with $300 \mathrm{ke} v$ self-ions.

Fig. 8. Comparison between (a) TRIM calculation of excess interstitials in Si after implantation with $100 \mathrm{keV}, 10^{16} \mathrm{As}^{+} / \mathrm{cm}^{2}$ with (b) cross-sectional TEM microzraph of a sample implanted with 
$185 \mu \mathrm{A} / \mathrm{cm}^{2}$. The depth scale in the micrograph matches the figure in (a). It should also be noted that a negative vilue for the interstitial excess denotes an excess vacancy concentration.

Fig. 9. Comparison of "substrate-aligned" and "near-surface aligned" spectra from a Si crystal implanted at $100 \mathrm{keV}$ with $4.5 \times 10^{14} \mathrm{Si}^{+} / \mathrm{cm}^{2}$. A rardom spectrum acquired while continuously rotating the sample is also shown.

Fig. 10. Angular scans about $<110>$ from strained and unstrained regions of a $100 \mathrm{keV}$ self-ion implanted Si sample. 
SCATTERING YIELD (counts)

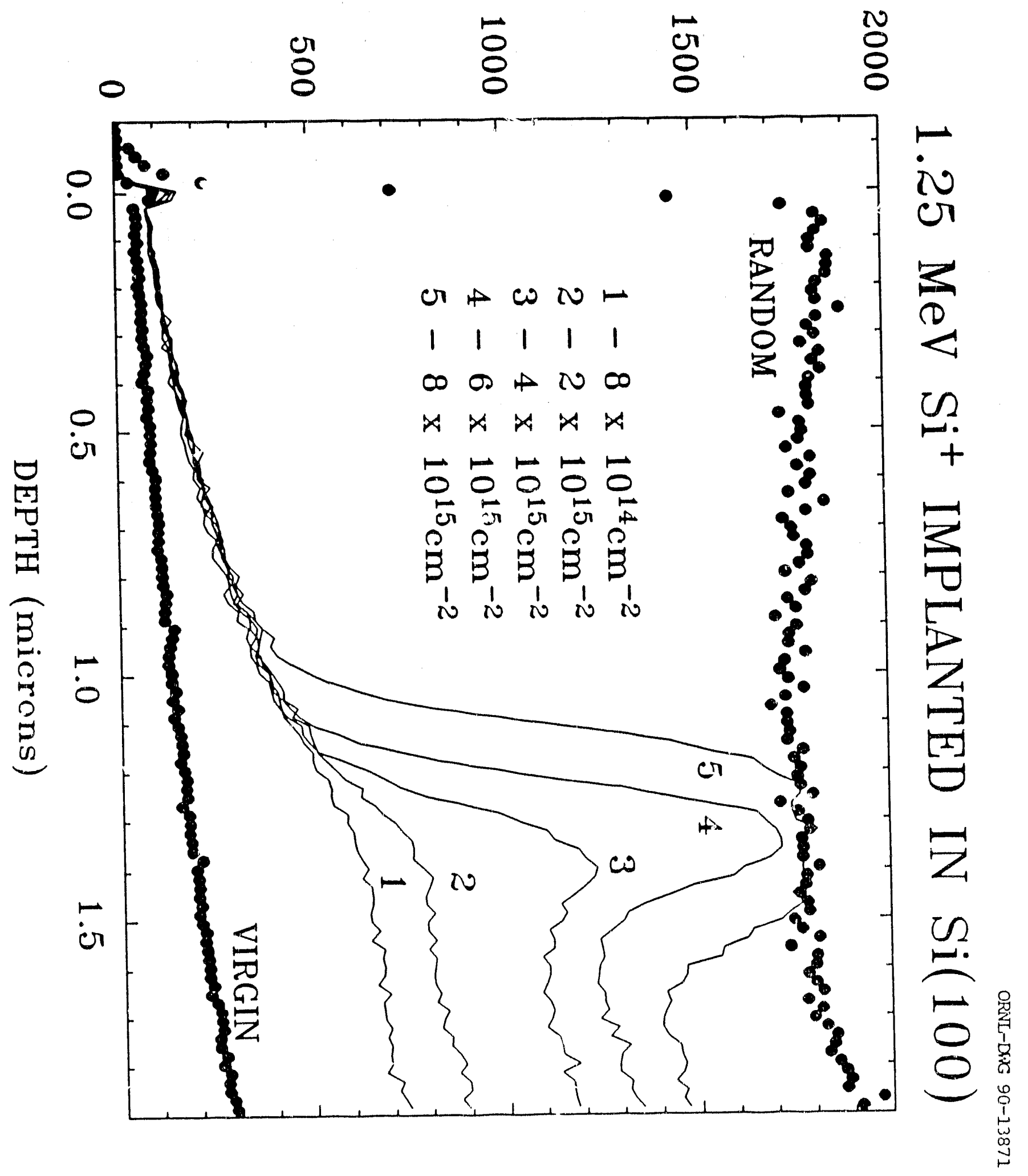




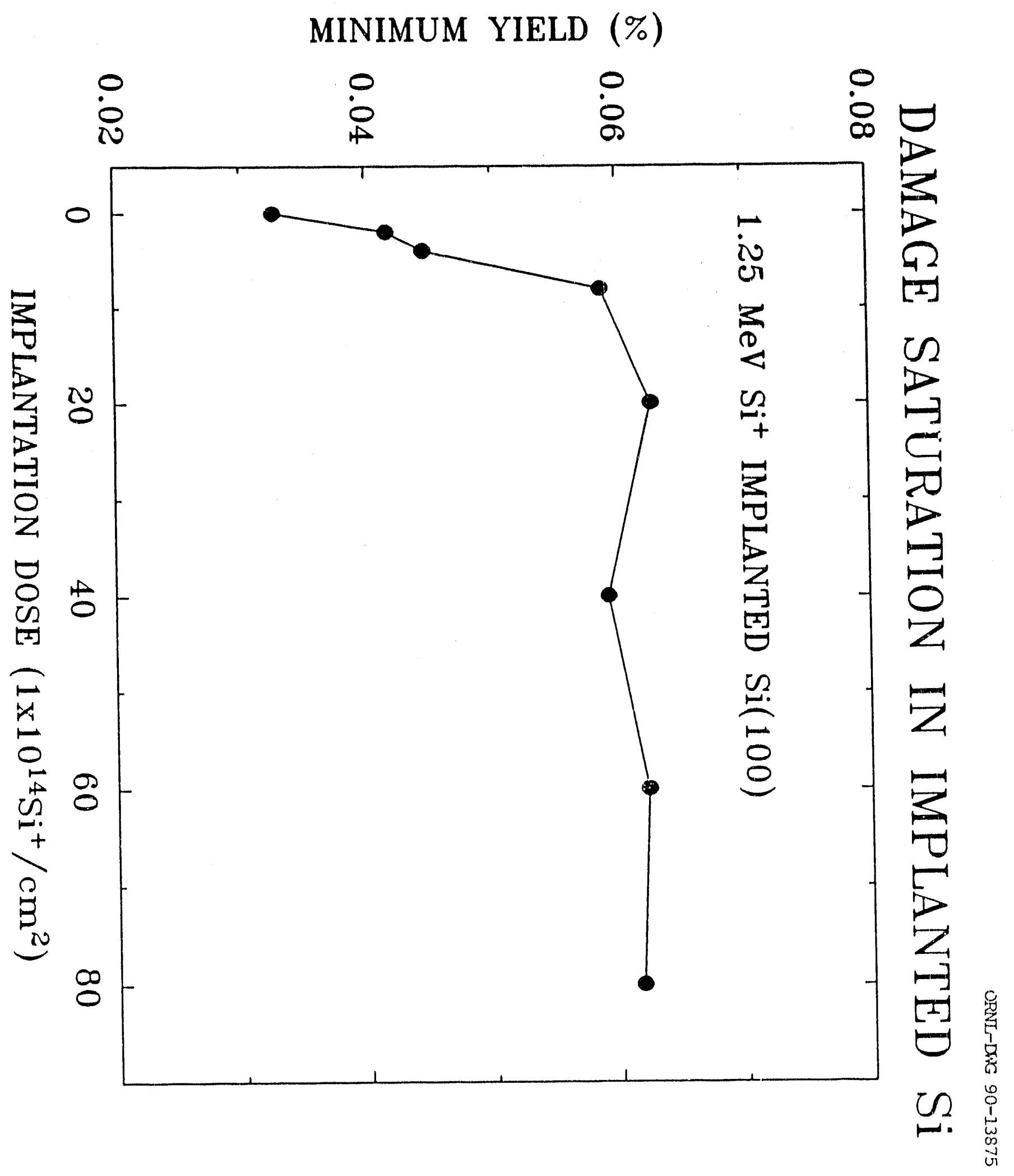

Fig. 


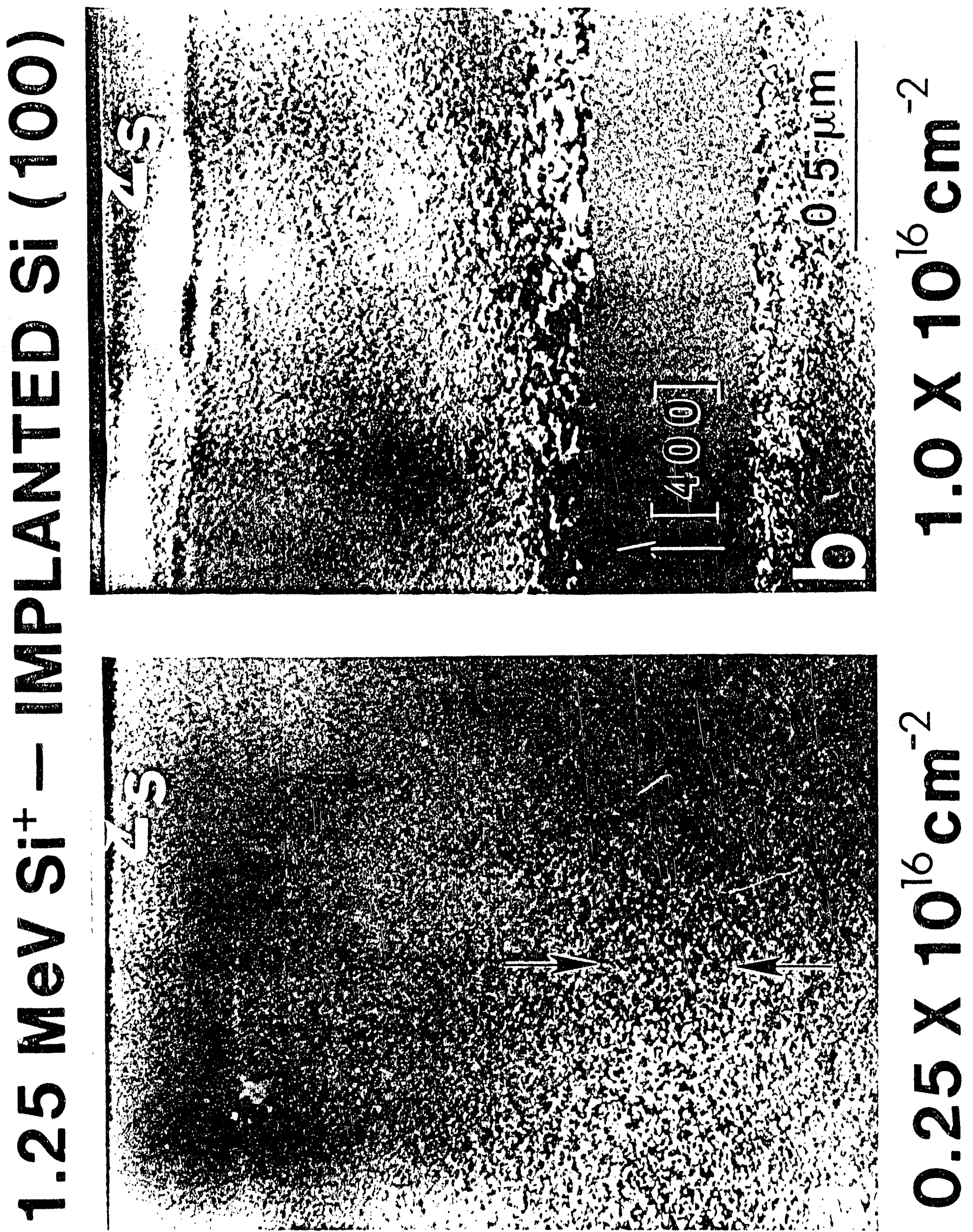




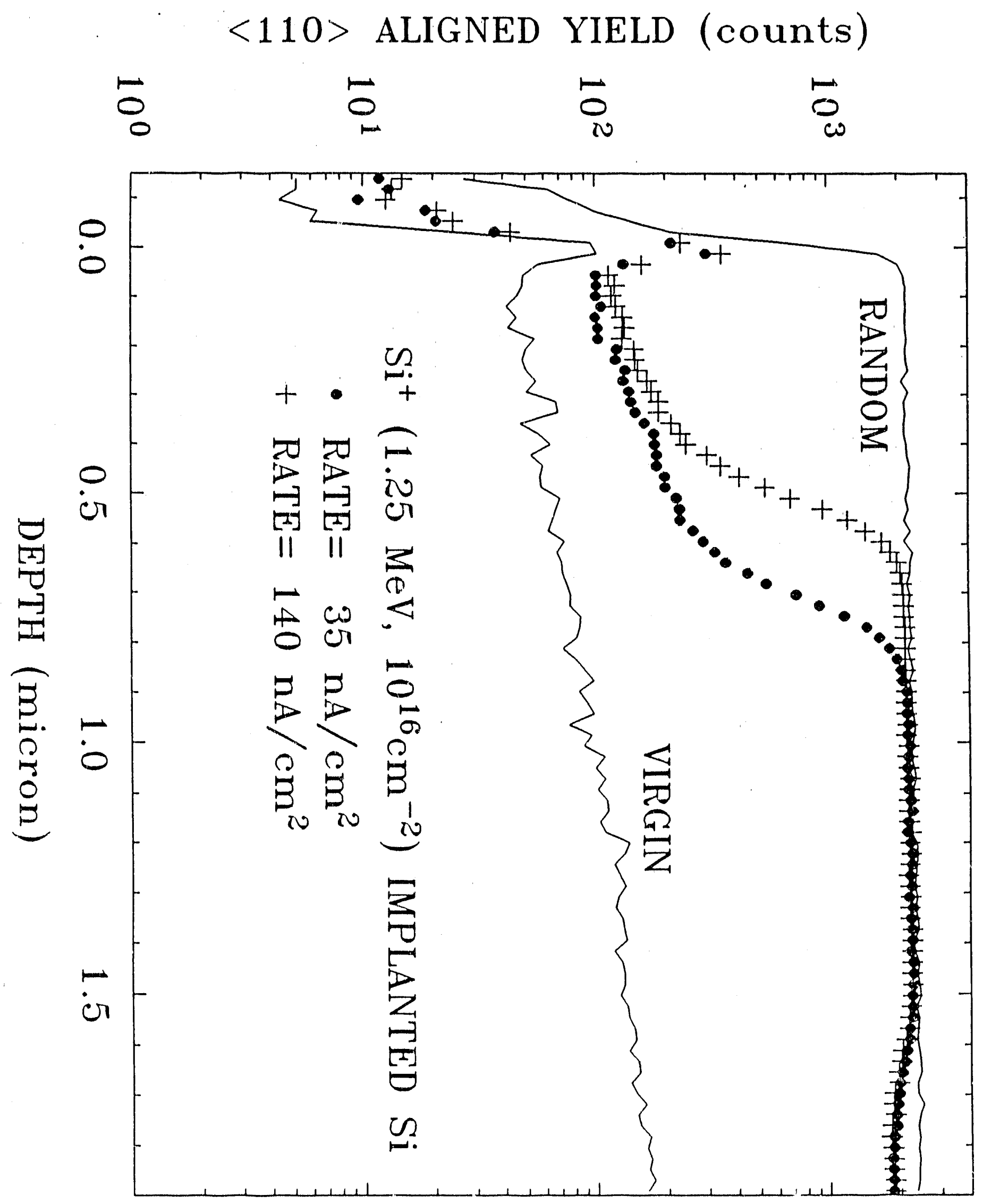

Fig. 4 


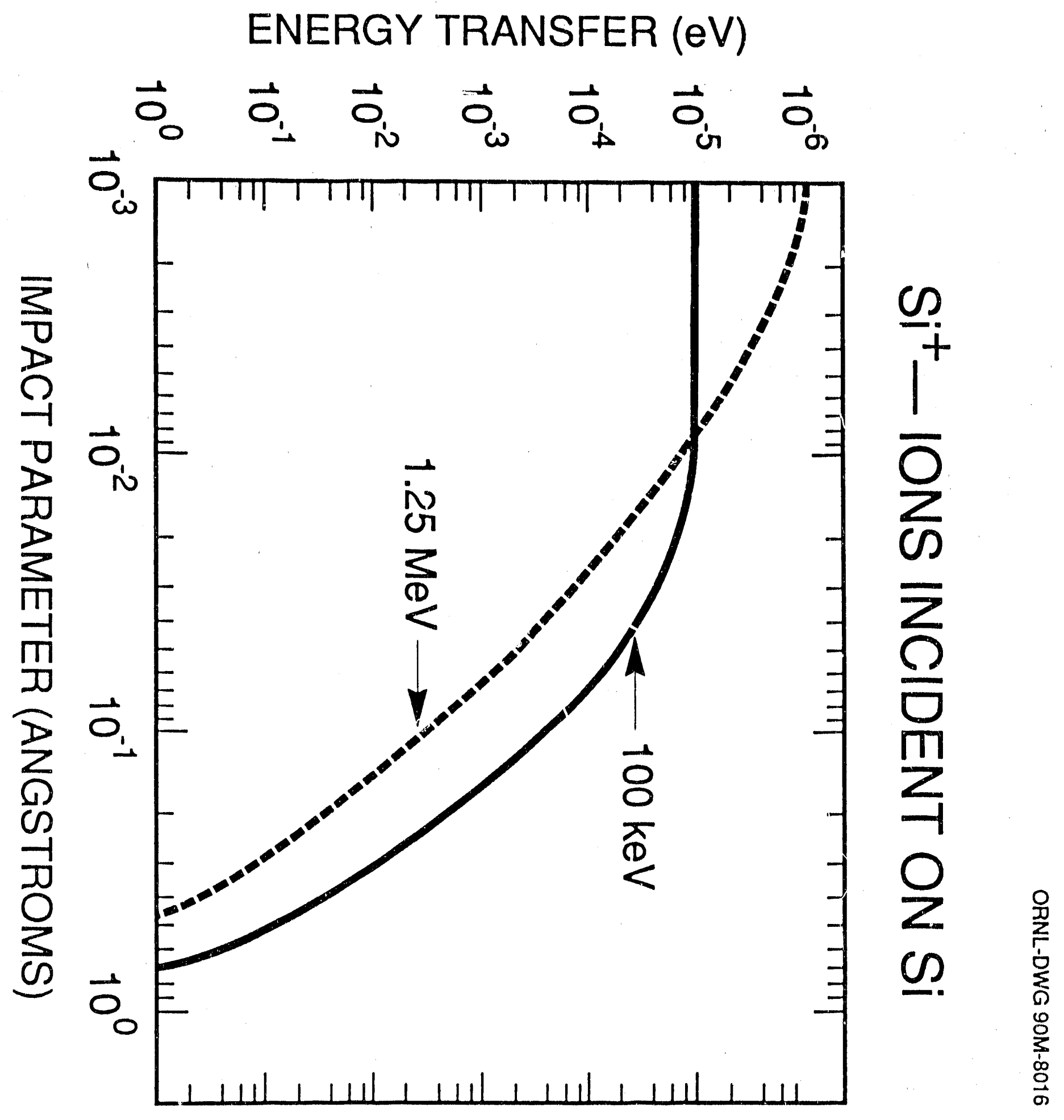




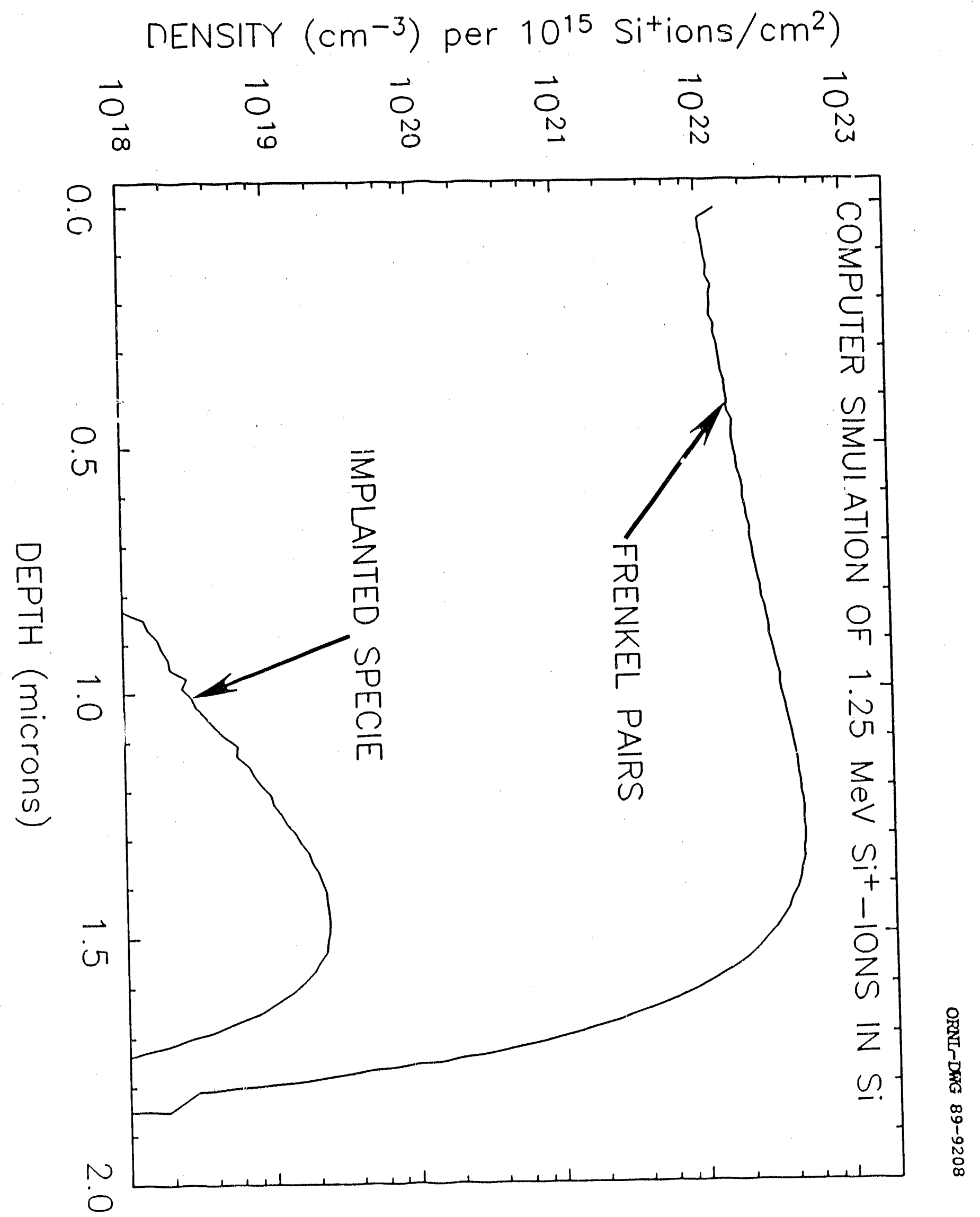

Fig. 6 


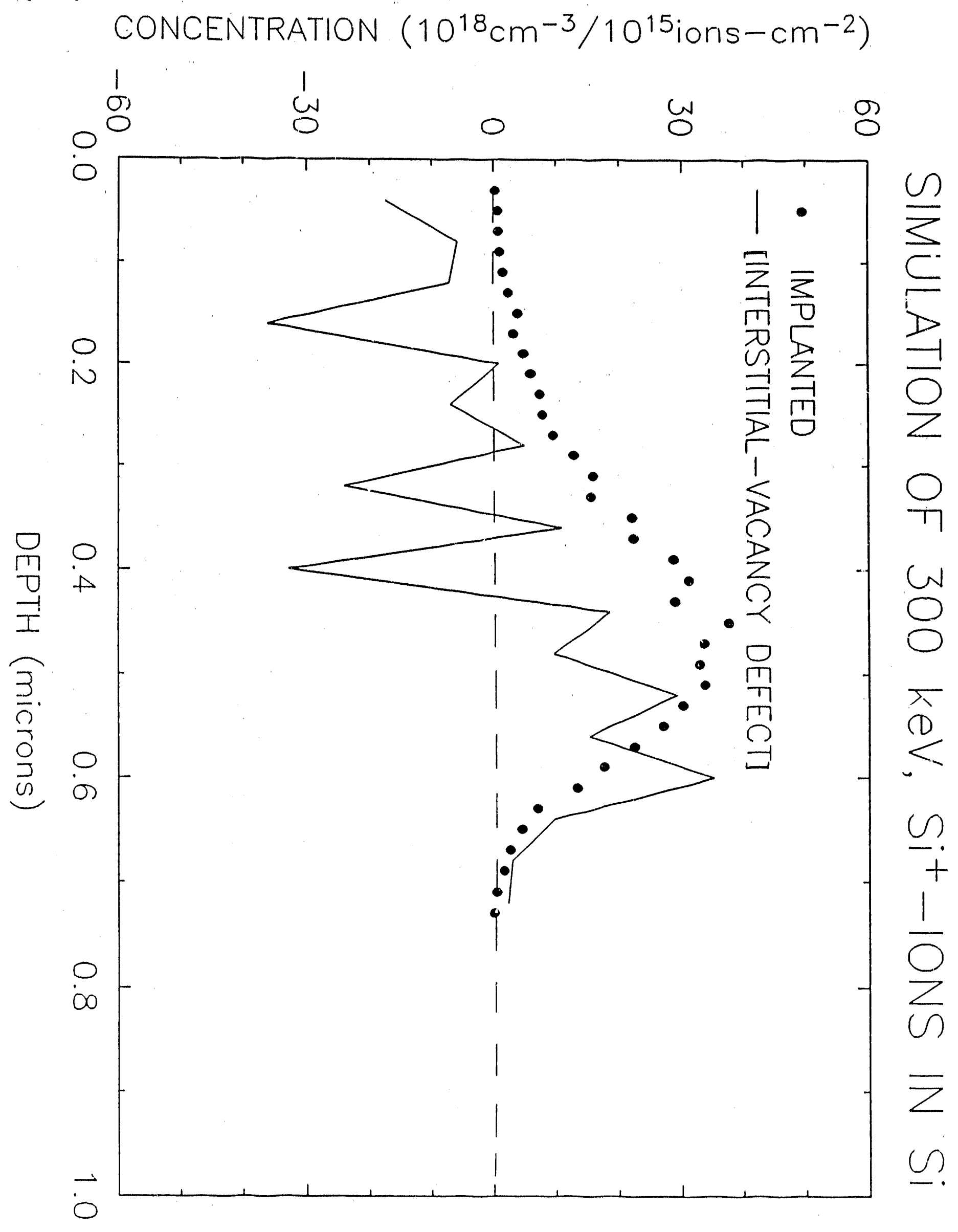

Fig. 7 

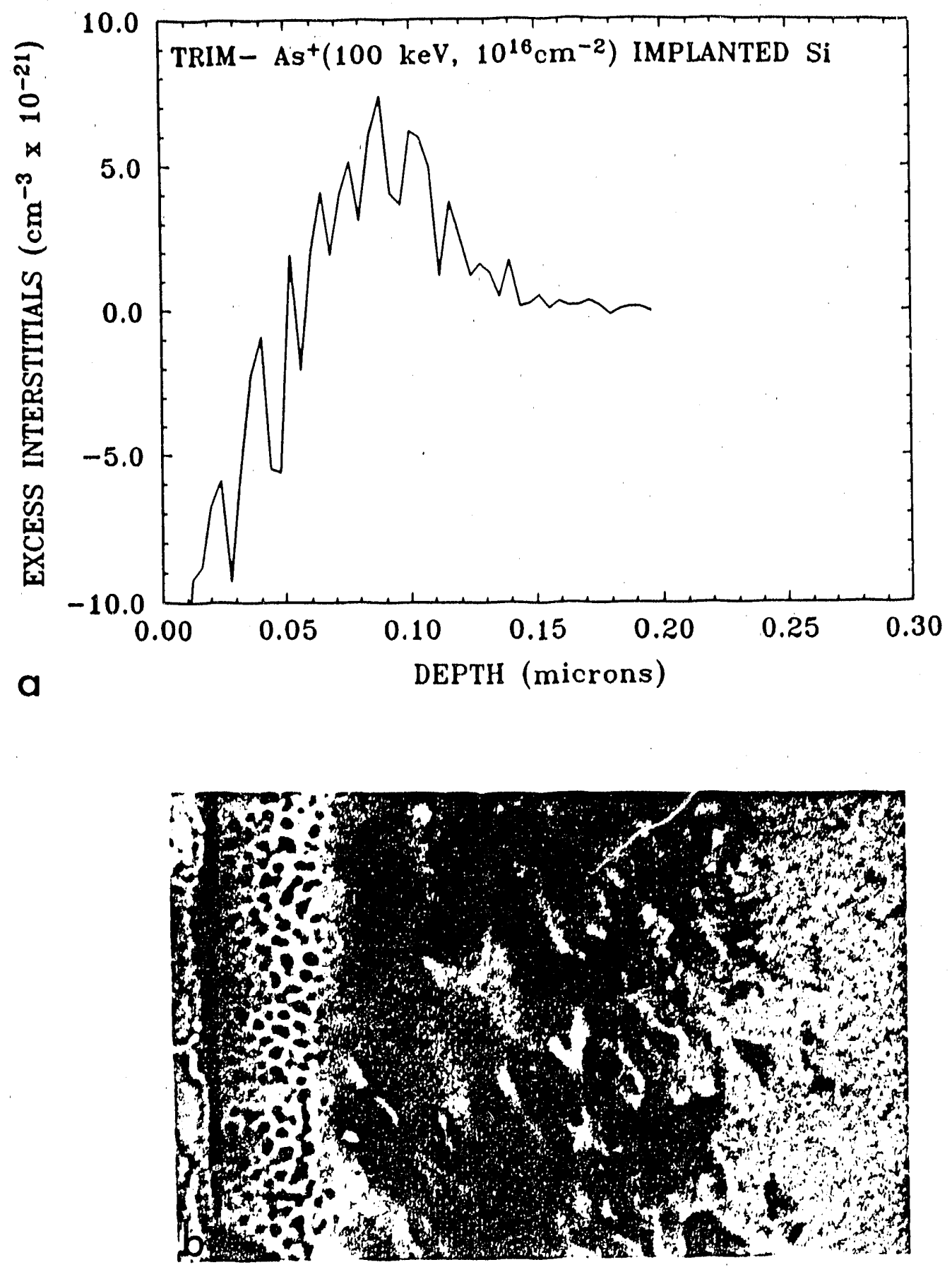

Fig. 8 


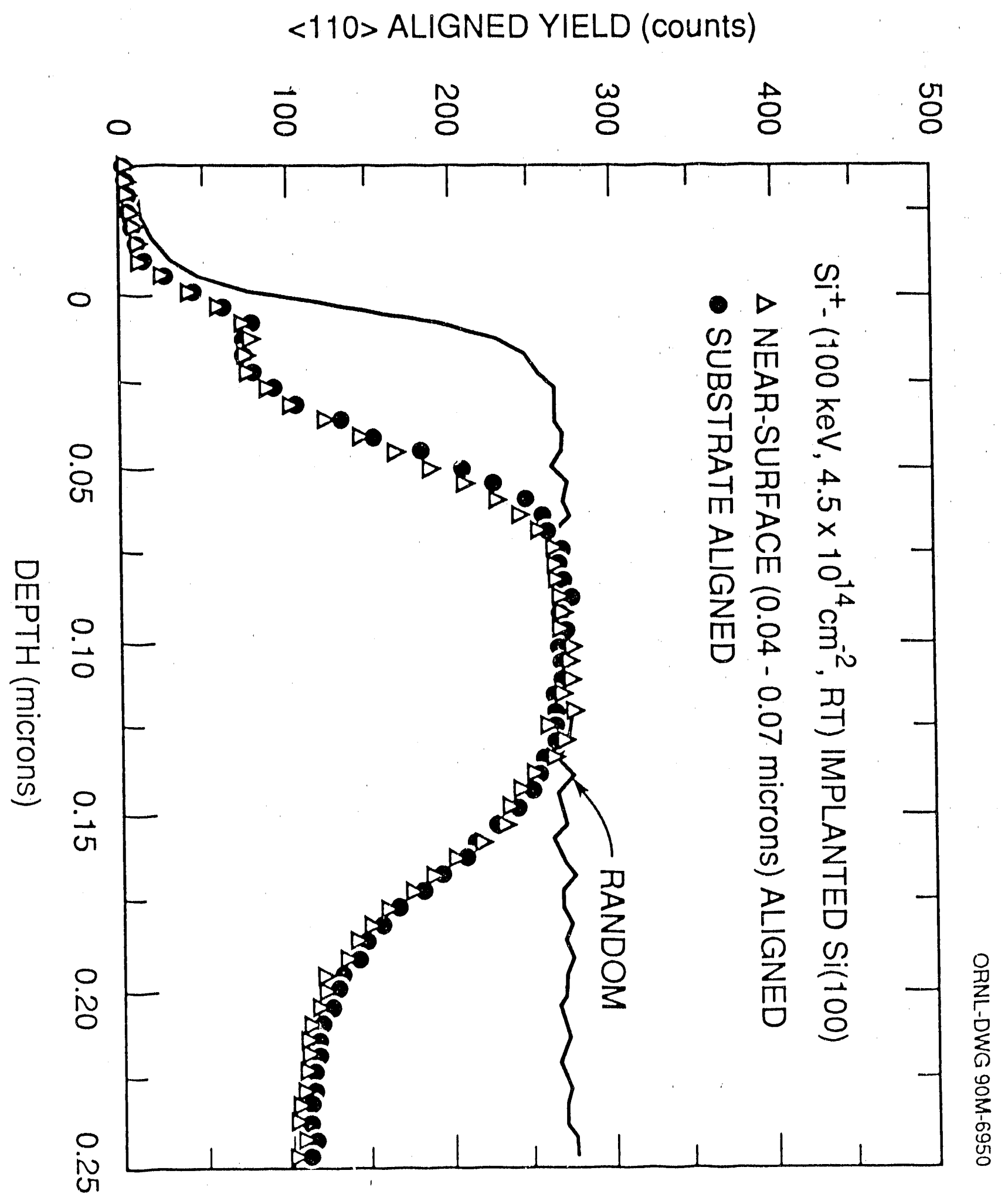

Fig? 


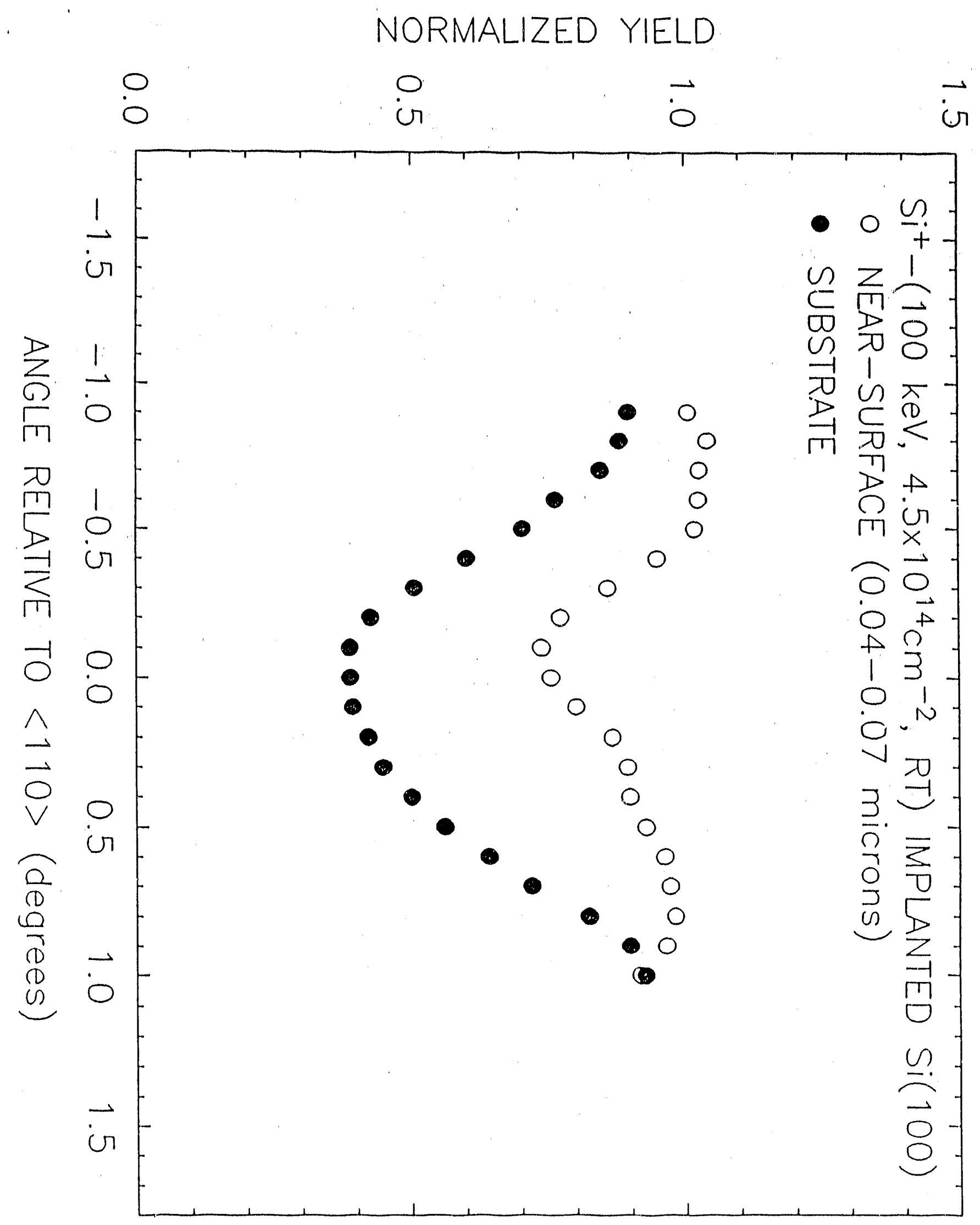

Fig. 10 

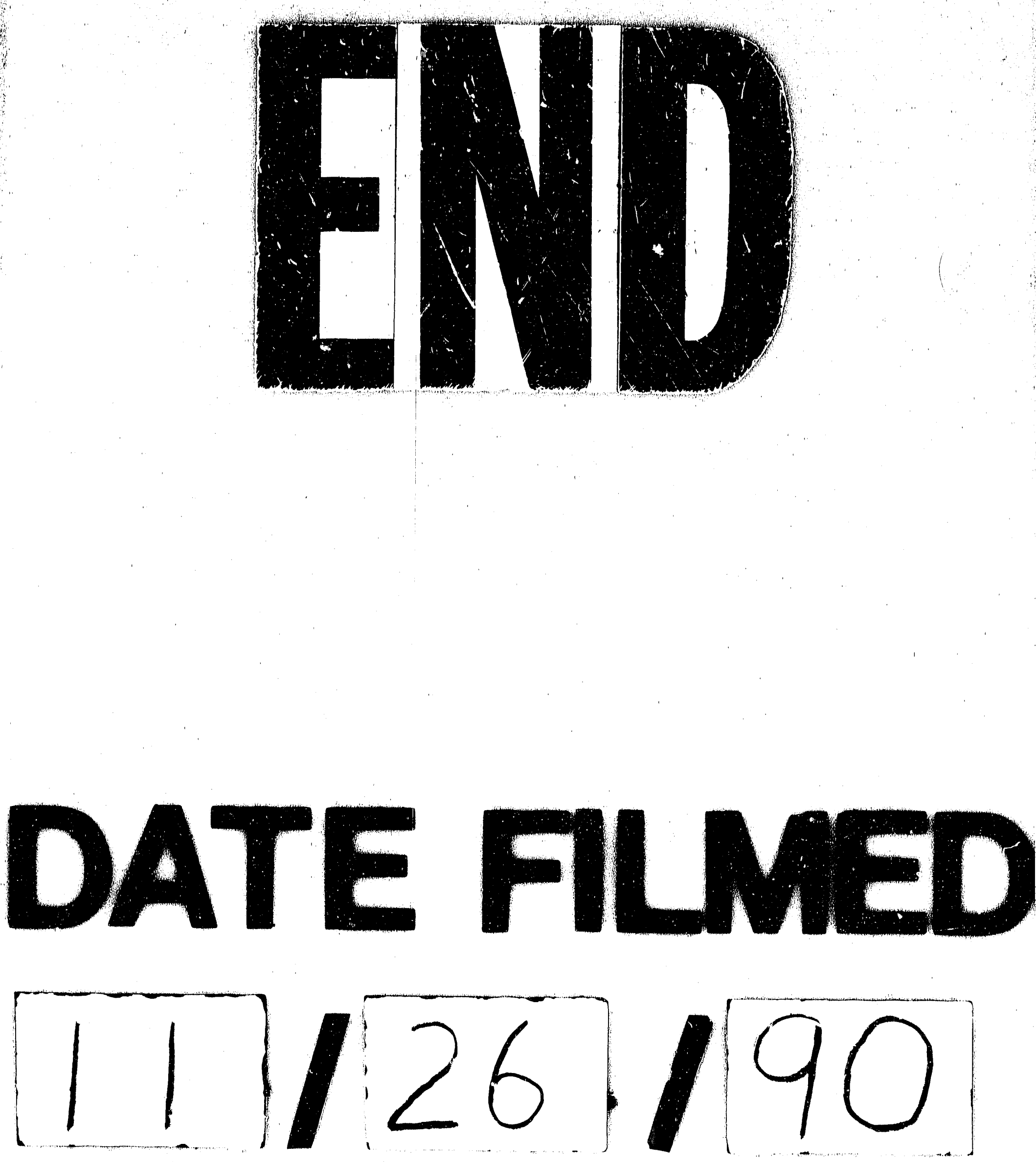